\title{
SiGe based Grating Light Valves: A leap towards monolithic integration of MOEMS
}

\author{
S. Rudra ${ }^{\text {a }}$, J. Roels ${ }^{\text {a }}$, G. Bryce ${ }^{\text {b }}$, L. Haspeslagh ${ }^{\text {b }}$, A. Witvrouw ${ }^{\text {b }}$, \\ D. Van Thourhout ${ }^{\text {a }}$ \\ ${ }^{a}$ Photonics Research Group, INTEC department, Ghent University - IMEC, Gent, Belgium \\ ${ }^{b}$ IMEC, Leuven, Belgium
}

The Grating Light Valve (GLV) is a microelectromechanical reflection grating. It operates on the principle of controlled diffraction of incident light due to electrostatic deflection of microbeams, thus producing bright and dark pixels in a display system. This unique approach offers significant advantages compared to other display systems in terms of speed, high optical efficiency, accuracy and ease of manufacturing. At the same time monolithic integration of MEMS on top of CMOS is getting more popular because CMOS-integrated MEMS exhibit less parasitics and have a reduced assembly and packaging cost over hybrid approaches. The relatively low deposition temperature $\left(\sim 450^{\circ} \mathrm{C}\right)$ of poly-SiGe compared to poly-Si $\left(\sim 800^{\circ} \mathrm{C}\right)$ makes poly-SiGe suitable for back end processing. Hence, in this work we report the fabrication and functioning of CVD poly-SiGe GLVs in terms of their response to the amplitude and frequency of the applied actuation voltage.

\section{Introduction:}

Grating Light Valve ${ }^{1,2}(G L V)$ display pixels are reflection type diffraction gratings. They consist of parallel microbeams moving up and down in response to an electric field forming a square well grating with changing phase as shown in Fig 1a. In the non-actuated state, coplanar beams behave like a mirror giving a specular reflection in the $0^{\text {th }}$ order. The pixel is then in the OFF state, with no light diffracted to the $\pm 1^{\text {st }}$ order. After actuation, the alternate movable beams are deflected downwards and the grating starts diffracting, which turns the pixel ON. At a deflection of $\lambda / 4$, the amount of light diffracted to the $\pm 1^{\text {st }}$ order reaches its maximum. Display systems made from this technology provide a huge improvement in contrast ratio, high resolution and brightness ${ }^{1,2}$ over other display systems. Recently it was reported that GLV based modulators can also be used in time of flight applications ${ }^{3}$.

The GLV microbeams we use are clamped-clamped (fixed-fixed) beams suspended in air over a substrate with the help of anchors on two far ends. They can be described by a spring capacitor model. The electrostatic force associated with the constant voltage drive mode is nonlinear and gives rise to the well known phenomenon of pull-in ${ }^{4,5}$. The electrostatically actuated beams collapse on the ground plane once the highest deflection exceeds approximately one-third of the airgap $\left(d_{0}\right)$. Hence in a GLV structure, the airgap between beams and the underlying ground substrate is designed in such a manner that $d_{0} / 3>\lambda / 4$. The actuation voltage is therefore always maintained well below the pull-in voltage, saving it from deterioration.

Another important issue is the integration of micro electro mechanical systems (MEMS) with $\mathrm{CMOS}^{6}$. In hybrid integration, separate chips for MEMS and IC are used which results in performance limiting parasitics because of the interconnections between them. These parasitics occur mainly from the size of the bondpads and from the long bonding wires. Comparatively, post processing of MEMS monolithically on top of CMOS can lead to increased functionality, performance, reliability and a higher miniaturization. However, post processing limits the thermal budget for the MEMS processing. Poly-SiGe provides the necessary mechanical properties and reliability required for MEMS applications at a significant lower temperature compared to poly-Si (i.e. deposition temperatures of $\sim 450^{\circ} \mathrm{C}$ instead of $\sim 800^{\circ} \mathrm{C}$ ). Hence, recently Poly-SiGe has attained serious attention in post processing MEMS above $\mathrm{CMOS}^{7}$. Monolithically integrated micro-mirror arrays ${ }^{8,9}$ are already reported. They can be used in applications such as video projection, adaptive optics, mask writers etc.

The purpose of this work is to show the functionality of poly-SiGe GLVs with different beam lengths. A clear length dependent change in resonance frequency of the devices is observed, as shown below. We will demonstrate that high switching speeds can be obtained and that a reasonable voltage dependent change in diffraction efficiency can be produced. 


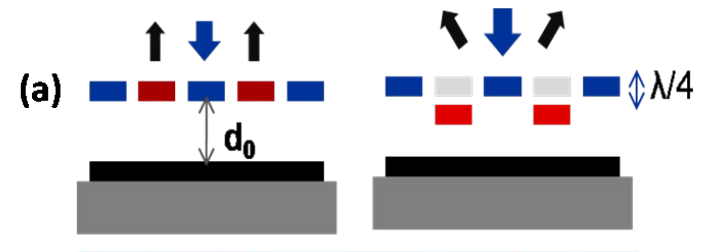

(b)

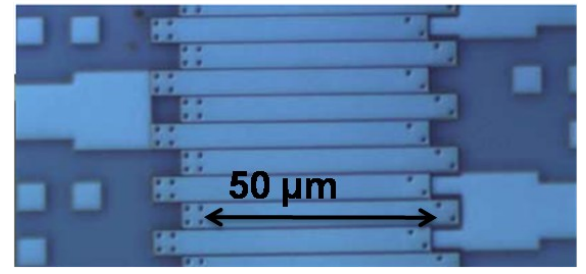

Fig.1. (a) Schematic representation of the working principle of a grating light valve, (b) Microscope view of GLV pixels consisting of two fixed and two movable beams.

\section{Fabrication and appearance of GLVs:}

The SiGe structural layer used in these devices is $300 \mathrm{~nm}$ thick. The CVD deposited structural SiGe layer was grown with a $\mathrm{SiH}_{4}$ : $\mathrm{GeH}_{4}$ flow ratio of $0.9: 1$ and $69 \mathrm{sccm} \mathrm{B}_{2} \mathrm{H}_{6}$ at a chuck temperature of $460^{\circ} \mathrm{C}(\sim$ wafer temperature of $450^{\circ} \mathrm{C}$ ). After roughness reduction of the SiGe layer by CMP, extra layers consisting of $5 \mathrm{~nm} \mathrm{SiC}$ and $30 \mathrm{~nm}$ AlCu were added. The AlCu layer is sputter-deposited at room temperature for high reflectivity and the $\mathrm{SiC}$ layer acts as a barrier layer to prevent diffusion between SiGe and Al. Finally the samples were released in vapor HF. Rutherford backscattering spectroscopy (RBS) data revealed a Ge concentration of about $78 \%$ in the SiGe layer ${ }^{10}$. Fig. $1 \mathrm{~b}$ shows the pixel configuration of the GLVs and the connection towards bondpads. A total of 16 pixels constitute a single GLV device. Three different beam lengths of $50 \mu \mathrm{m}, 100 \mu \mathrm{m}$ and $200 \mu \mathrm{m}$ were fabricated with a pitch of $4.8 \mu \mathrm{m}$ and a fill factor of $93 \%$. The airgap between the beams and the substrate was maintained at $0.6 \mu \mathrm{m}$, allowing GLV operation over the whole visible spectrum.

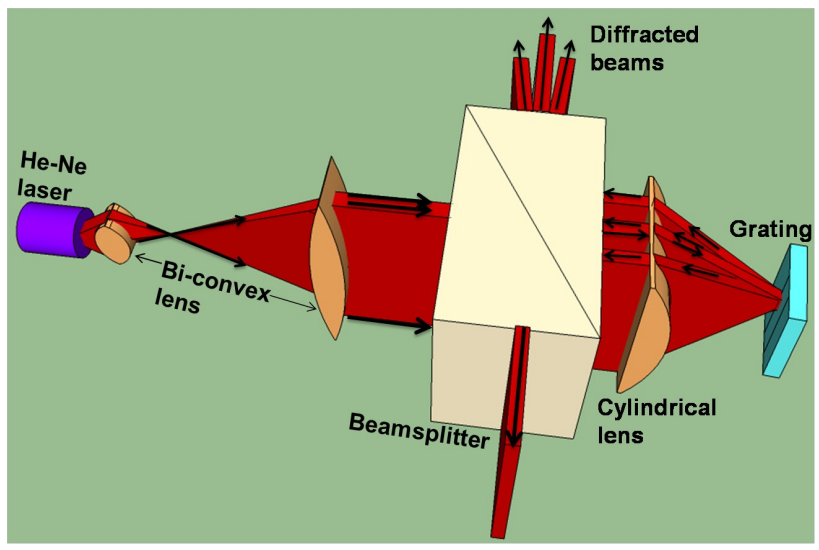

Fig. 3. GLV characterization set-up.

\section{Experimental characterization system:}

Fig. 3 shows the experimental set-up used for characterization of the GLVs. The two convex lenses in the front act as a collimator. The cylindrical lens focuses the light as a horizontal line with uniform intensity on the center of the microbeams. The beamsplitter helps in separating the incoming and outgoing light. A photodiode in series with an electrical spectrum analyzer gives the intensity of the diffracted light as a function of amplitude and frequency of the applied actuation voltage.

\section{Results and Discussion:}


To ensure a safe operation, the pull-in voltage of different GLVs with varying beam lengths was estimated. COMSOL Multiphysics ${ }^{11}$ (based on finite element method analysis) was used to calculate the pull-in voltage of the devices using a fixed-fixed beam model. As can be seen from Fig. $4 \mathrm{~b}$ the pull-in voltage for $50 \mu \mathrm{m}, 100 \mu \mathrm{m}$ and $200 \mu \mathrm{m}$ beams vary widely, which can be verified easily using an analytical spring capacitor model as reported in literature ${ }^{4,12}$. A Young's modulus of $143 \mathrm{GPa}$ and a density of $4725 \mathrm{Kg} / \mathrm{m}^{3}$ was assumed for the simulation and the theoretical calculations ${ }^{11}$.

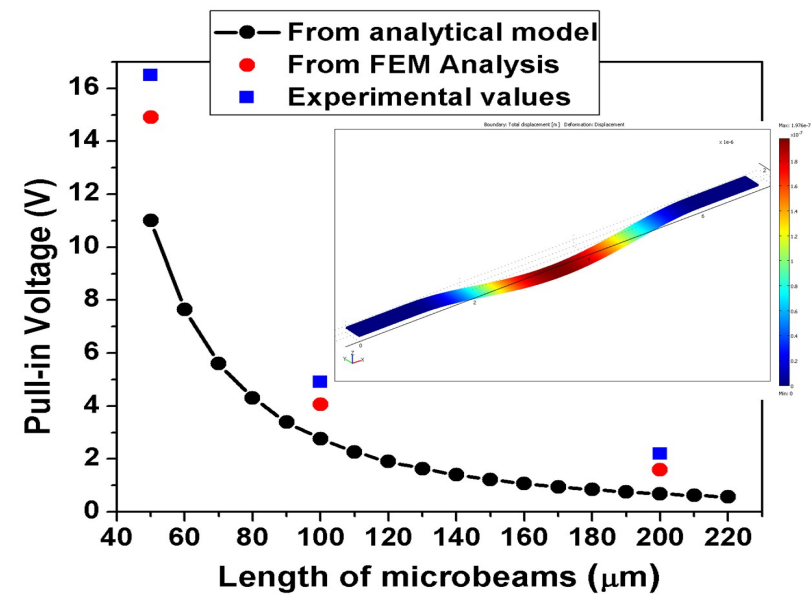

Fig. 4. Comparison of pull-in voltage values with change in beam length as found from finite element analysis using COMSOL, an analytical model ${ }^{12}$ and experiments. The inset shows a typical COMSOL simulation of bending of a $50 \mu \mathrm{m}$ long and $4.5 \mu \mathrm{m}$ wide fixed-fixed electrostatically actuated SiGe beam with an airgap of 0.6 $\mu \mathrm{m}$.

Once the safe operating range was determined, the GLV devices were thoroughly characterized. Proper understanding of the dynamic characteristics of electrostatically actuated microbeams are of utmost importance for the MEMS devices. GLV microbeams can be well described by a forced, damped, harmonic oscillator for which the response time decreases with increasing resonant frequency and damping coefficient. We measured the resonant frequency to estimate the switching speed of the devices. The change in optical intensity under DC actuation was also monitored to determine the contrast of the present device. To find the frequency response of the devices, a small sinusoidal signal $\left(\mathrm{V}_{\mathrm{AC}}\right)$ was used in combination with a larger $\mathrm{DC}$ voltage $\left(\mathrm{V}_{\mathrm{DC}}\right)$ to modulate the beams around a stable deflected distance. This creates a force with two different harmonics of the applied signal but the $1^{\text {st }}$ harmonic dominates largely over the $2^{\text {nd }}$ one because of the small amplitude of the sinusoidal signal :

$F \propto\left[V_{D C}+V_{A C} \cos \omega t\right]^{2} \rightarrow\left[\left(V_{D C}^{2}+\frac{V_{A C}^{2}}{2}\right)+2 V_{D C} V_{A C} \cos \omega t+\frac{V_{A C}^{2}}{2} \cos 2 \omega t\right.$

The small signal frequency response of the devices is shown in Fig. 5 where a clear increase in resonance frequency with decreasing length of microbeams can be observed. Using a spring capacitor model theoretical ${ }^{13,14}$ resonance frequencies of $703 \mathrm{KHz}, 175 \mathrm{KHz}$ and $44 \mathrm{KHz}$ were found respectively for the 50,100 and $200 \mu \mathrm{m}$ beams which is in relative agreement with the experimentally determined values of $920 \mathrm{KHz}, 255$ $\mathrm{KHz}$ and $124 \mathrm{KHz}$. The reason behind the deviation between experimental and theoretical values may be related to: (1) the application of a relatively high DC voltage causes initial bending and gives rise to a tensile stress inside the beams which was not taken into account in the theoretical calculations,or (2) an uncertainty in the estimated Young's Modulus and density of the poly-SiGe material. We also determined experimental $Q$ factors of $1.6,3.2$ and 5.2 respectively for the 200,100 and $50 \mu \mathrm{m}$ beams. These relatively small Q's can be attributed to the air damping in open atmosphere. 


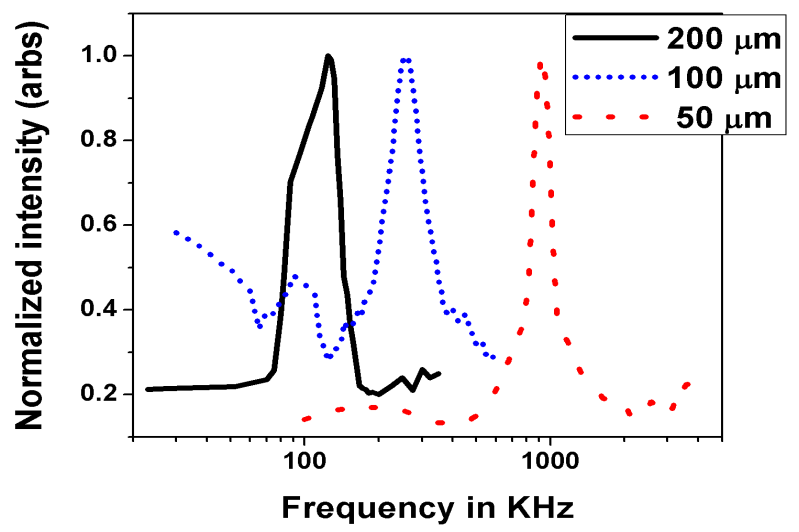

Fig. 5. Small signal frequency response of GLVs with 50,100 and $200 \mu \mathrm{m}$ long beams.

The response of the GLVs to a DC voltage is shown in Fig. 6. A clear change in the intensity of the $1^{\text {st }}$ order diffracted light with increasing actuation voltage can be observed. Whereas for the $100 \mu \mathrm{m}$ beams the change is less prominent and the dark state intensity is high, the $50 \mu \mathrm{m}$ beams show an excellent overall behavior. This might be related to the intrinsic stress in the structural SiGe/SiC/Al stack. This stress could not be measured accurately due to stress changes in the underlying Si-oxide layer during the deposition of the structural layer. However, as the $50 \mu \mathrm{m}$ beams are flat and the $100 \mu \mathrm{m}$ and longer beams seem slightly buckled, the intrinsic stress is probably compressive. It is expected that the deficient behavior of the long beams can be improved by introducing a tensile stress in the beams.

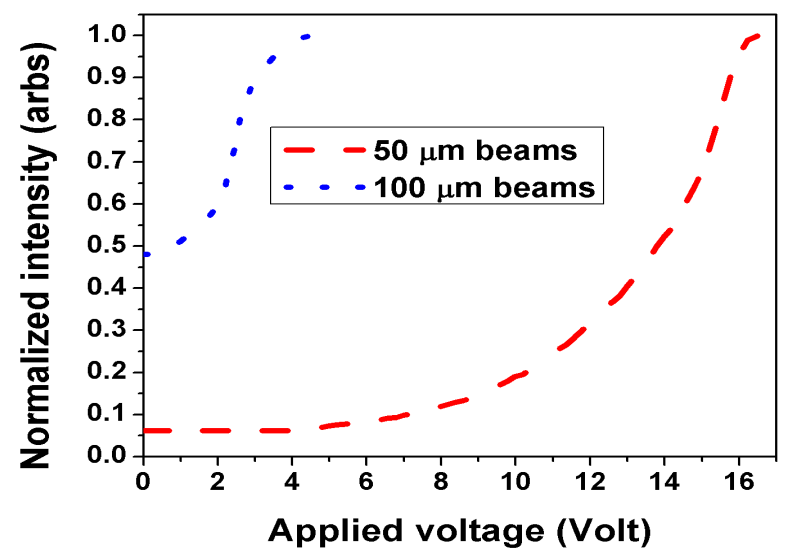

Fig. 6. Change in $1^{\text {st }}$ order diffraction intensity with actuation voltage for two different GLVs.

\section{Conclusion:}

We have demonstrated the operation of a poly-SiGe based GLV for the very first time. GLVs of $50 \mu \mathrm{m}$ showed a good tuning behavior with a resonance frequency of $920 \mathrm{KHz}$. Our next goal is to implement a residual tensile stress in the microbeams which will improve the switching speed and the dark state intensity level.

\section{References:}

1. D. Bloom, "The Grating Light Valve: Revolutionizing Display Technology," SPIE Proceedings, 3013, 165171, Projection Displays III Symposium, 1997

2. Trisnadi et al, Photonics West 2004, Micromachining and Microfabrication symposium, Paper 5348-05

3. J. Roels et al, Photonics Technology Letters, 20 (2008), 1827.

4. G.M. Rebeiz, RF MEMS: Theory, Design and Technology, John Wiley \& Sons, p. 21-31, 2003.

5. D. Peroulis et al, IEEE Trans. On Microwave theory and techniques, 51 (2003), 259-270. 
6. R. Jablonski, M. Turkowski and R. Szewczyk, Recent Advances in Mechatronics, Springer Berlin Heidelberg (2007), 521-525.

7. A. Witvrouw, Scripta Materialia, 59 (2008), 945-949.

8. M. Gromova, et al, Proc. IEEE MEMS 2007, p. 759-762

9. L. Haspeslagh, J. De Coster, et al, , Proc. IEDM 2008, pp. 655-658 (2008).

10. G. Bryce et al, Proc. ECS 2008, ECS Transactions, Vol 16, Issue 10, 353-364 (2008)

11. M. Uncuer, et al, Proc. COMSOL conference 2007, p. 439

12. S. Chowdhury et al, J. Micromechanical Systems, 15 (2005), 756-763.

13. W.T. Thomson, Theory of vibration with applications, Englewood Cliffs, NJ: Prentice Hall, 1981

14. K. Wang et al, J. Microelectromechanical Systems, 9 (2000) 347-360. 